\title{
The faint intergalactic-medium red-shifted emission balloon: future UV observations with EMCCDs
}

\author{
Gillian Kyne ${ }^{a}$, Erika T. Hamden ${ }^{a}$, Nicole Lingner ${ }^{a}$, Patrick Morrissey ${ }^{a}$, Shouleh Nikzad $^{b}$, D. \\ Christopher Martin ${ }^{a}$ \\ ${ }^{a}$ California Institute of Technology, 1200 E. California Blvd, Pasadena, CA 91125 USA. \\ ${ }^{b}$ Jet Propulsion Laboratory, California Institute of Technology, Pasadena, CA 91109 USA.
}

\begin{abstract}
We present the latest developments in our joint NASA/CNES suborbital project. This project is a balloon-borne UV multi-object spectrograph, which has been designed to detect faint emission from the circumgalactic medium (CGM) around low redshift galaxies. One major change from FIREBall-1 has been the use of a delta-doped Electron Multiplying CCD (EMCCD). EMCCDs can be used in photon-counting (PC) mode to achieve extremely low readout noise $\left(\mathrm{i} 1 \mathrm{e}^{-}\right)$. Our testing initially focused on reducing clock-induced-charge (CIC) through wave shaping and well depth optimisation with the CCD Controller for Counting Photons (CCCP) from Nüvü. This optimisation also includes methods for reducing dark current, via cooling and substrate voltage adjustment. We present result of laboratory noise measurements including dark current. Furthermore, we will briefly present some initial results from our first set of on-sky observations using a delta-doped EMCCD on the 200 inch telescope at Palomar using the Palomar Cosmic Web Imager (PCWI).
\end{abstract}

Keywords: astronomical instrumentation, EMCCD, UV, dark current, CIC, photon counting

\section{INTRODUCTION}

FIREBall-2 is a balloon-borne ultraviolet (UV) multi-object spectrograph. It is designed to discover and map faint emission from the circumgalactic medium (CGM) of low redshift galaxies $(\mathrm{z} \sim 0.7)$. This balloon is an upgraded version of FIREBall-1, which has flown twice before in 2006 and 2009.1,2 The spectrograph has been redesigned to significantly increase the field of view, throughput, and number of targets per observation, ${ }^{3-5}$ yielding a factor of 30 increase in overall sensitivity. This should also provide multiple detections of the CGM in emission for the first time at UV wavelengths. CNES is providing the spectrograph, gondola, and gondola flight support team. Currently, construction of all components is complete and we are in the final alignment stages with continuous testing. FIREBall-2 has upgraded the science detector since its inception, where we will be demonstrating a relatively new technology using an electron multiplying charge coupled device (EMCCD). EMCCDs were chosen for this mission due to their photon-counting (PC) abilities; a mode used to achieve extremely low readout noise $\left(<1 \mathrm{e}^{-}\right)$. This detector has been developed at JPL to provide UV sensitivity using a delta-doping process, which is discussed further by Nikzad ${ }^{6,7}$ and Hoenk. ${ }^{8}$ The process yields a device with $100 \%$ internal quantum efficiency (QE), limited only by surface reflections. Anti-reflection coatings to minimize this reflectance bring the QE of the FIREBall-2 device up to $>65 \%$ from 200-210 nm. FIREBall-2 uses an e2v CCD201-20 in photon counting mode. This device is normally operated in frame transfer mode, however, the FIREBall-2 instrument makes use of the entire $1 \mathrm{~K} \times 2 \mathrm{~K}$ array.

Further author information: (Send correspondence to Gillian Kyne)

E-mail: gkyne@caltech.edu, Telephone: +1 6263954296

High Energy, Optical, and Infrared Detectors for Astronomy VII, edited by Andrew D. Holland, James Beletic, Proc. of SPIE Vol. 9915, 991507 - (c) 2016 SPIE · CCC code: 0277-786X/16/\$18 · doi: 10.1117/12.2232879 


\subsection{Electron Multiplying CCDs}

Electron Multiplying CCDs (EMCCDs) are a relatively recent development in CCD technology. ${ }^{9-11}$ They can can be used in "photon-counting" mode with extremely low readnoise. EMCCDs operate similarly to a standard $\mathrm{CCD}$, but have a second serial register containing additional pixels, where R2 is replaced with a high voltage (HV) clock. As individual electrons pass through the multiplication register they are multiplied via impact ionization. The exact multiplication gain achieved is a stochastic process controlled by the maximum voltage of the HV clock. Each individual transfer has a relatively low probability of multiplication $(<2 \%)$, but when an electron passes through all 604 multiplication pixels (in the case of an e2v CCD201-20), the final number of electrons generated at the end pixel can be quite large. The testing described in this work uses an electron multiplication (EM) gain of around $1000 \mathrm{e} / \mathrm{e}$.

The major advantage of using EM gain is that it increases the signal from a single photo-electron to a value much larger than the on-chip amplifier readnoise. This process means that single photon events can be detected by a threshold process described in greater detail by Tulloch ${ }^{12}$ and Harpsøe. ${ }^{13}$ In general, pixels with counts greater than about 5 times the readnoise are considered to have had 1 event. Pixels with counts less than this threshold are considered to have zero events. There are some complications as a result of this process. Not all electrons in the device will be amplified above this $5 \sigma$ threshold. The multiplication process is random, so some fraction of real events will not be multiplied enough to be visible. The ratio of EM gain to readnoise provides an estimate for the number of events which are not counted because of low amplification, as described by Daigle. ${ }^{14}$ Additional sources of noise come from clock-induced-charge (CIC) and dark current. In a standard CCD, when cooled below $-85^{\circ} \mathrm{C}$ and with normal trapezoidal or square clocks, dark current and CIC will be lower than the readout noise (assuming a few electrons), and thus undetectable. However, an EMCCD will amplify these sources of noise in the same way as photo-electrons. Dark current can be reduced by operating in inverted mode and by cooling the device further. Recent work exploring dark current at lower temperatures show evidence for a possible lower limit ${ }^{15,16}$ but most applications operate at $-85^{\circ}$ and so the behaviour below this temperature is not well explored. Furthermore, cold operation of the CCD can lead to a significant reduction in charge transfer efficiency (CTE).

We present the results of our dark current testing in both an engineering grade and a delta-doped EMCCD. Low noise is achieved through wave shaping and well depth optimization (to reduce CIC) and via cooling (for low dark current). Our group has been working on the optimization of these devices using a Nüvü Controller and a test set-up at Caltech. We are operating our devices in NIMO (non-inverted mode operation) and use cooling to achieve lower dark current. We have adjusted our clocking to reduce CIC through wave shaping and reduced image clock wells. Daigle ${ }^{17}$ has reported great success in minimizing CIC through the use of sinusoidal and triangular shaped clocks. Both shapes reduced CIC by a factor of 10 at the lowest readout frequencies. It was also found that clock shaping did not exhibit increased CIC with decreased pixel readout speed. Testing continues to find an optimized clocking scheme to maintain low dark current and CIC, without loss of charge and hence reduced device QE/throughput.

\subsection{Detector noise testing}

This paper will describe our testing of the FIREBall-2 flight detector. Our main goal is to produce a clocking configuration that will achieve the lowest noise by minimizing readnoise, dark current, and CIC. We have made improvements to our readout electronics to reduce our readnoise, and will be exploring slower HV clocking. We have reduced CIC with lower parallel and serial wells, and clock shaping. Our dark current testing is ongoing, however, we have been investigating dark rate as a function of cooling and substrate voltage. All of this testing has been carried out using a version V2 Nüvü controller. In the coming months we will be exploring a larger parameter space for noise reduction using a version 3 controller, which provides us with four readout clock speeds, 1, 2, 10, and 20MHz. The version 3 controller has a $5 \mathrm{~ns}$ waveform resolution for improved clock shaping, versus $10 \mathrm{~ns}$ on the version 2 .

The majority of our testing has been conducted on a number of engineering grade CCD201-20 devices procured directly from e2v. We have begun testing on a number of delta-doped, AR-coated devices (processed at JPL); some of these devices have been packaged by JPL and e2v. In Section 2 we describe our laboratory test set-up. In Section 3 we present results from noise testing of two engineering grade devices, and a number of delta-doped 
EMCCDs. In Section 4 we discuss some preliminary on-sky data taken with a delta-doped EMCCD on Palomar Cosmic Web Imager (PCWI) and Section 5 describes our noise performance targets for the FIREBall-2 flight.

\section{FIREBALL-2 LABORATORY TEST SET-UP}

The laboratory test set-up uses a custom Printed Circuit Board (PCB) for both laboratory and flight. This board is used as a rigid harness that holds the sensor in place, and also serves as a vacuum interface for the test dewar. The board includes RC filters located close to the sensor pins to reduce any residual noise ripples in the signal trace. Grounding plates, as well as grounding paths, are used around the traces to give a low inductance path for the current at high frequencies, to further quiet any noise in the transmission lines.

FIREBall uses a Nüvü v2 CCD Controller for Counting Photons (CCCP), ${ }^{14}$ which was selected for its versatile waveform shaping ability and fast clocking speeds. The readout electronics for the v2 controller operate at 10 MHz; the v3 provides more readout options as discussed above. The Nüvü controller is connected to the PCB with a SAMTEC EQCD high speed coax cable.

The EMCCD is secured on both sides of the PCB by a gold plated copper (cold-) clamp and cooled with a CryoTel MT mechanical cooler (Sterling Engine) from Sunpower. The device cold-clamp is connected to the cryo-cooler by a cold-finger, which makes a second contact to a charcoal getter. The charcoal getter is used to maintain vacuum pressure when the system is in flight and vacuum can't be maintained by a turbo pump. It's important that the charcoal getter is held below $-165^{\circ} \mathrm{C}$ to achieve optimal pumping. The pressure rise measured in the laboratory is about $1 \times 10^{-6}$ Torr/day. Vacuum pressure is maintained at $1 \times 10^{-6}$ Torr using a Varian V-250 turbo pump, when operating in the laboratory. Pressure and temperature are monitored at all times during testing. A Lakeshore 218 controller is used in combination with multiple DT-670 silicon diode sensors, to measure temperature at a number of surfaces. A second proportional-integral-derivative (PID) Omega controller has also been installed. This unit uses a PT100 RTD sensor placed in close proximity to the device. The sensor has been calibrated (preliminary calibration) in our laboratory against these silicon diodes. The Omega controller also operates two $10 \mathrm{~W}$ heaters that maintain a constant device temperature for all testing. This is especially important when photon-counting as the HV clock is extremely sensitive to temperature changes. Internal dewar pressure is monitored using a 354 series Ionization Vacuum Gauge. The ion-gauge sensor emits light and is turned off during dark current and noise measurements.

\section{CCD201-20 NOISE MEASUREMENT AND PERFORMANCE}

We have constructed a laboratory set-up to efficiently perform noise testing of an e2v CCD201-20. This testing has been carried out on number of engineering grade and delta-doped devices. The delta-doping process is carried out at JPL where some of these devices are also packaged, see more by Nikzad. ${ }^{18}$ Our initial testing consisted of optimizing our waveform scripting confirm that the device could image and photon-count. The first stage of noise testing consisted of minimizing CIC. The Nüvü software is flexible such that we can select an image array size that provides us with horizontal overscan pre- and post-image readout. This way we can calculate our parallel CIC from the image area.

\section{$3.1 \mathrm{CIC}$}

$\mathrm{CIC}$, or spurious noise, is caused by impact ionization of holes as they move through the $\mathrm{Si} / \mathrm{SiO}_{2}$ interface during pixel clocking. ${ }^{19}$ In a normal CCDs, CIC is not apparent due to readnoise levels of a few electrons. In EMCCDs, CIC is also subject to impact ionization and so can be counted as an event, indistinguishable to a photo-electron. This is why it is important to minimize this false charge. Methods of reducing CIC include the following:

- Faster clock speeds produce less CIC

- Substrate voltage - more holes are generated/present in the clocks during inversion

- Percentage clock overlap

- Clock well voltage amplitude 
Our work to reduce CIC was done in two stages. The initial waveform script, provided by Nüvü, used trapezoidal/square shaped image clocks with $50 \%$ overlap, a clock swing of -5 to $7 \mathrm{~V}$, and a voltage substrate of $4.5 \mathrm{~V}$ also known as inverted mode operation (IMO). We found that imaging clocks such as these can produce a reasonable image, but high noise. With this configuration we measured CIC at 0.055 events/pix/frame. In non-inverted mode (NIMO) at 0V, the parallel CIC dropped to 0.005 events/pix/frame. First we investigated the impact of reducing the voltage swing of each imaging clock, until we reached a point where charge transfer was lost. As indicated above, the initial well depth for our testing was $12 \mathrm{~V}$. We experimented with changing both maximum and minimum values, including a staggered maximum value for successive clocks. We found this to be the best approach to retain acceptable charge transfer. The final working range for these wells was 10-11V. Lower well depths reduced the CIC to 0.01-0.02 events/pix/frame at a substrate voltage of $4.5 \mathrm{~V}$. A voltage substrate of $0 \mathrm{~V}$ with lower wells reduced parallel CIC to 0.002 events/pix/frame. The next stage of testing consisted of clock shaping, from square to sinusoidal waves. We saw a significant reduction in CIC, with an improved measurement of 0.001 events/pix/frame at both 0 and $4.5 \mathrm{~V}$ substrate voltage. We saw no degradation of CTE using these clock shapes at any voltage substrate.

A similar procedure was carried out on the serial clocks to achieve lower serial CIC. We measured an initial serial CIC of 0.02 events/pix/frame at $4.5 \mathrm{~V}$, and 0.05 events/pix/frame at $0 \mathrm{~V}$. These measurements were taken at a well depth of $12 \mathrm{~V}$. Reducing the serial wells to $10 \mathrm{~V}$ reduced serial CIC to 0.002 events/pix/frame at $4.5 \mathrm{~V}$. Further improvement was found by wave shaping and we measured 0.0007 events/pix/frame at $4.5 \mathrm{~V}$ and 0.0005 events/pix/frame at $0 \mathrm{~V}$. Our work through wave shaping and well depth optimization has reduced total CIC to 0.0017 events/pix/frame for IMO, and 0.0015 events/pix/frame for NIMO. Table 1 summarizes these findings. We have also measured CIC with a delta-doped device (dark current will be discussed in Section 3.2). These results are not presented in this paper, but are comparable to those found with the engineering grade device.

Table 1. Table summarizing CIC measurements for inverted (4.5V substrate voltage) and non-inverted (0V substrate voltage) operating modes, both before, during, and after optimization. ${ }^{20}$

\begin{tabular}{c|c|c|c|c} 
& & Initial Value & Reduced Well Depth & Shaped \& Reduced Wells \\
\hline \multirow{2}{*}{ IMO } & Parallel & 0.055 & 0.02 & 0.001 \\
& Serial & 0.02 & 0.002 & 0.0007 \\
& Total & 0.075 & 0.022 & 0.0017 \\
\hline \multirow{2}{*}{ NIMO } & Parallel & 0.005 & 0.002 & 0.001 \\
& Serial & 0.05 & 0.005 & 0.0005 \\
& Total & 0.055 & 0.007 & 0.0015
\end{tabular}

\subsection{Dark Current}

Dark current is a well known source of noise in silicon based devices. ${ }^{21}$ This thermal noise is generated in a conventional CCD, and at low temperatures is masked by the readnoise. However, this same thermal noise present in an EMCCD will be amplified in the same way as a photo-electron. Dark current can therefore be measured in photon-counting mode. Part of our detector noise testing has focused on measuring CIC (see Section 3.1), in both IMO and NIMO. Lower CIC can be achieved in NIMO, and lower dark current in IMO. Our primary focus has been NIMO, as we can reduce dark current by cooling. This dark current testing has been carried out over a range of temperatures, between -85 and $-125^{\circ} \mathrm{C}$. The device temperature is monitored via a silicon diode on the cold-clamp. Previous testing has shown that the front of the device is $7^{\circ} \mathrm{C}$ warmer than the back of the cold-clamp. A non-functioning device was used to record temperature at the front of the device.

During our initial testing of an e2v CCD201-20 engineering grade device we found a lower level of dark current (almost half) in the storage (masked) area, compared to the image area. One possible reason for this 


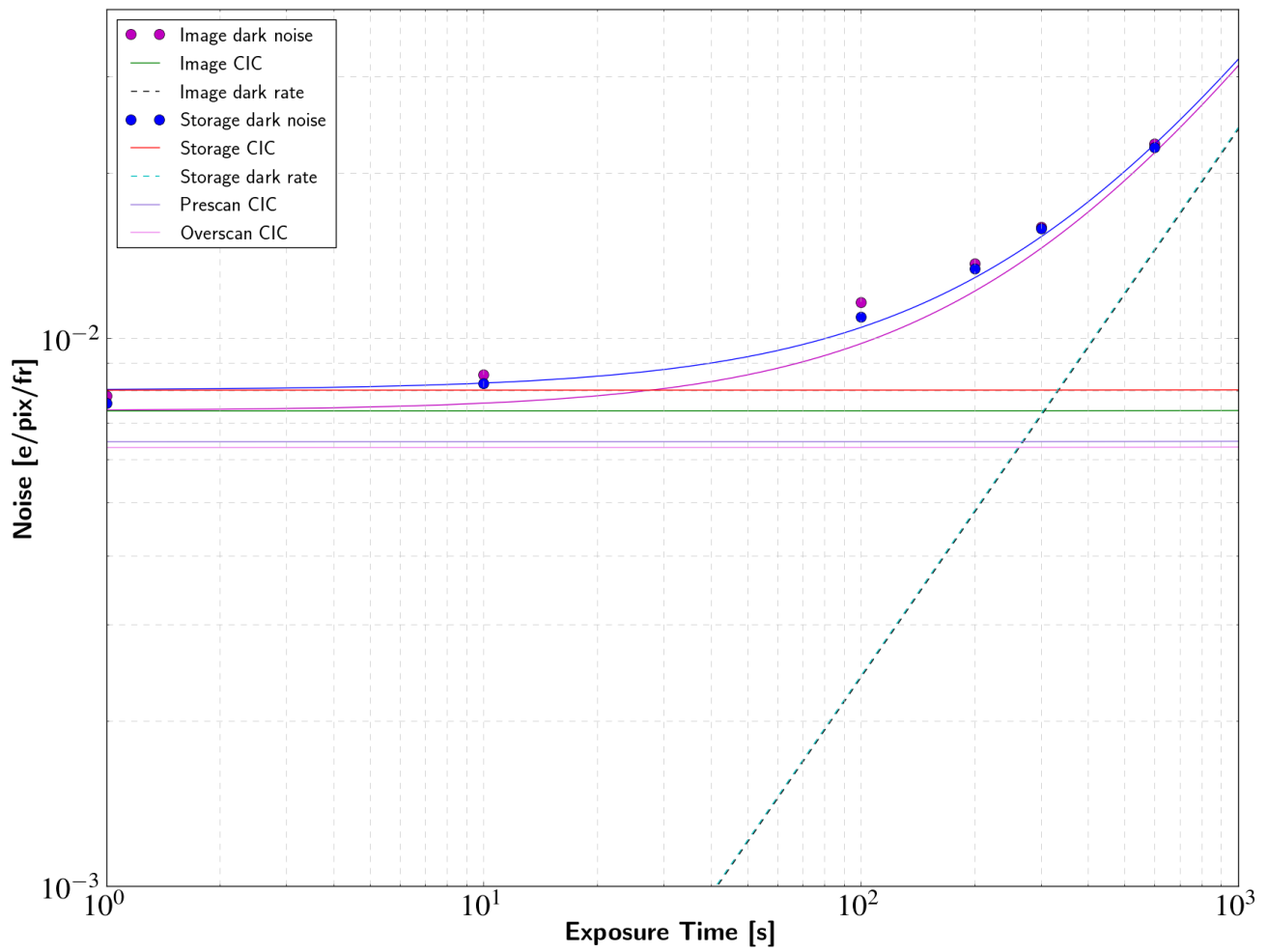

Figure 1. This is an example of a dark current measurement from an e2v CCD201-20 delta-doped device, wafer 7 device 17. The delta-doping process takes place at JPL. This data set was taken at $-108^{\circ} \mathrm{C}$. The dark purple and blue lines represent the image and storage area dark current, respectively. The green, red, pink, and purple plot the image, storage, overscan, and prescan CIC, respectively. The dotted black and cyan lines indicate the least-squares line fit for the CIC subtracted data in the image and storage areas. The temperature measurement was taken from the back of the CCD cold-clamp and is normally $7^{\circ} \mathrm{C}$ cooler than the CCD surface.

could be due to passivation of the back surface by the aluminium storage shield. Dark current measurements began once we established the new waveform script that was capable of imaging with reduced CIC. When this testing began we powered on the Nüvü controller while the device was at room temperature. This is due to a property of this powering up called "injected charge" where we found that the dark current was higher if the controller was powered on while cold. Since our testing began over 12 months ago we have implemented clocking of the dump gate. This clears the serial register prior to a new exposure and readout. More detail of this initial testing can be found by Hamden. ${ }^{20}$

Dark current measurements were carried out by taking a series of exposures (5-10 images) at increasing exposure times. The Nüvü software has a live viewing feature, and live statistics that are updated when a change is made to the waveform script. We use this to find a value for the high voltage clock that gives an EM gain of 1000e/e. Once the data is acquired the number of events per frame is calculated using a $5 \sigma$ threshold, and averaged for exposure time. The image readout includes an over- and prescan region, either side of the image area. CIC events are measured from the zero second exposure in the dark current set, and this value is removed from subsequent exposure times to measure dark current. Dark current was measured for both storage and image of the engineering grade device, and the same analysis was used for the delta-doped device to find an overall average for the image area. This method is used for each temperature and voltage substrate measured. The dark current rate is measured using a least-squares line fit to the plot of events versus exposure time. See Figure 1 for an example of this plot.

For both engineering grade and delta-doped e2v CCD201-20 devices, we found dark current decreased with temperature until approximately $-110^{\circ} \mathrm{C}$. We have found that below this temperature we reach a 


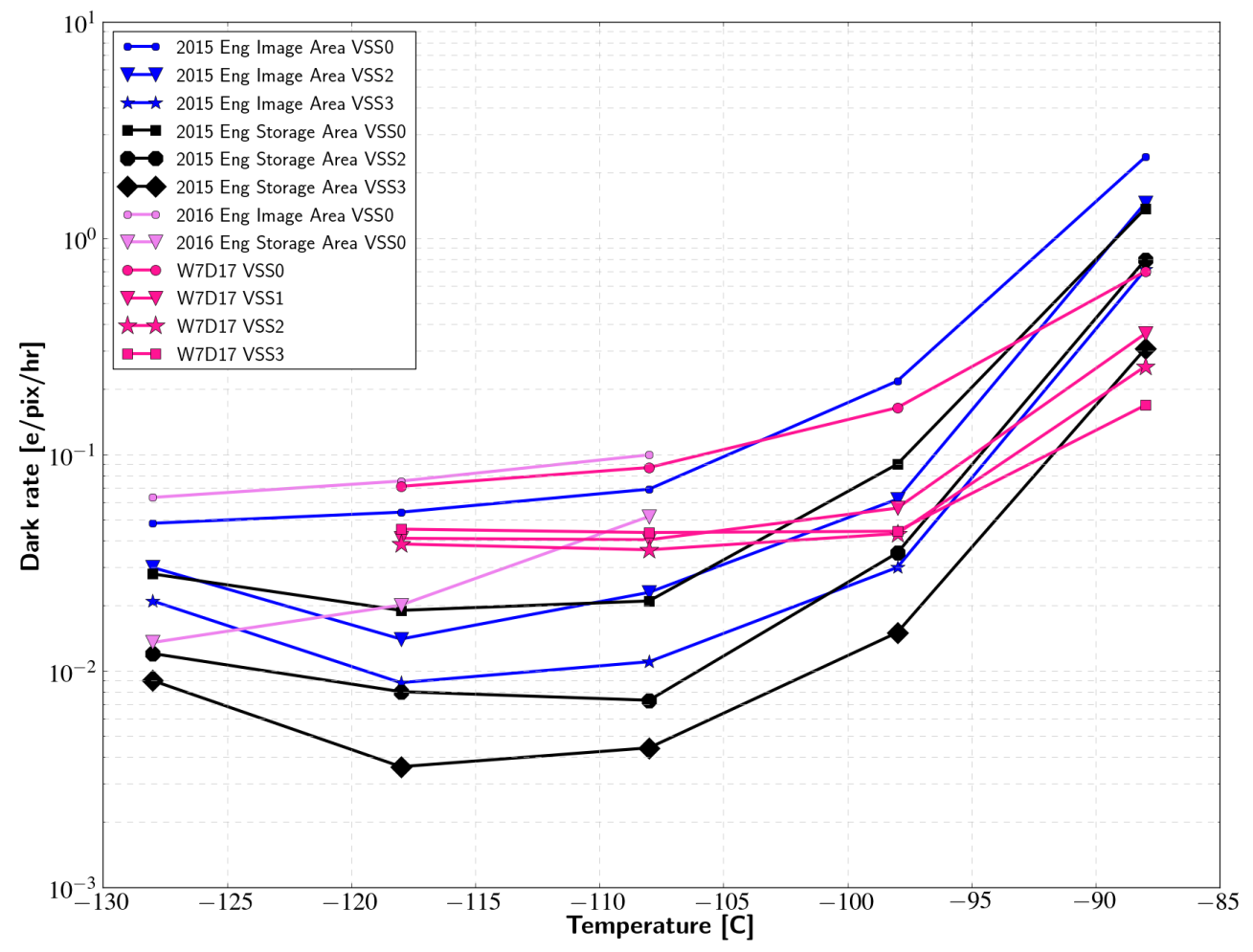

Figure 2. Dark current rates as a function of temperature. This plot shows dark current rates for two engineering grade devices and a delta-doped device. Image area and storage area are plotted for engineering devices. Colours represent different devices. Part of the test also varied voltage substrate.

plateau where lower temperature does not signifcantly reduce dark current, and in some cases potentially introduces more noise from poor charge transfer. We have also found that moving into inversion (higher voltage substrates) reduces the dark current, as expected. The plateau remains with changing voltage substrate at a lower level; these plots can be found in Figure 2. Our dark current testing is on-going, and part of this will be a further investigation of the plateau. The plateau could, in part, be a combination of a low level light leak and spurious charge from a cosmic ray hit, which cannot be removed in analysis. Our best dark current measurement for the masked area of an engineering grade device was 0.004 events $/ \mathrm{pix} / \mathrm{hr}$ at $-118^{\circ} \mathrm{C}$, and a voltage substrate of $3 \mathrm{~V}$. The delta-doped device measured a rate of 0.04 events $/ \mathrm{pix} / \mathrm{hr}$ at $-118^{\circ} \mathrm{C}$, but at a voltage substrate of $2 \mathrm{~V}$. 


\section{DELTA-DOPED EMCCD AT PALOMAR}

We brought a delta-doped device to the P200 inch telescope on Palomar Mountain in May 2016, where we were awarded four nights on the Cosmic Web Imager (CWI). CWI is a semi-facility instrument at Palomar.

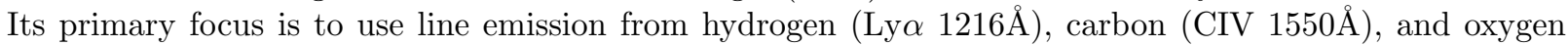
(OVI $1036 \AA$ ) to detect and map diffuse gas surrounding galaxies and quasars at redshifts $1.5<\mathrm{z}<7$. Measuring the strength, and mapping the extent of this emission will provide a greater understanding of the interactions between forming and evolving galaxies and their surroundings. ${ }^{22}$ Some images of the CWI instrument, and an example of data can be found in Figure 3.
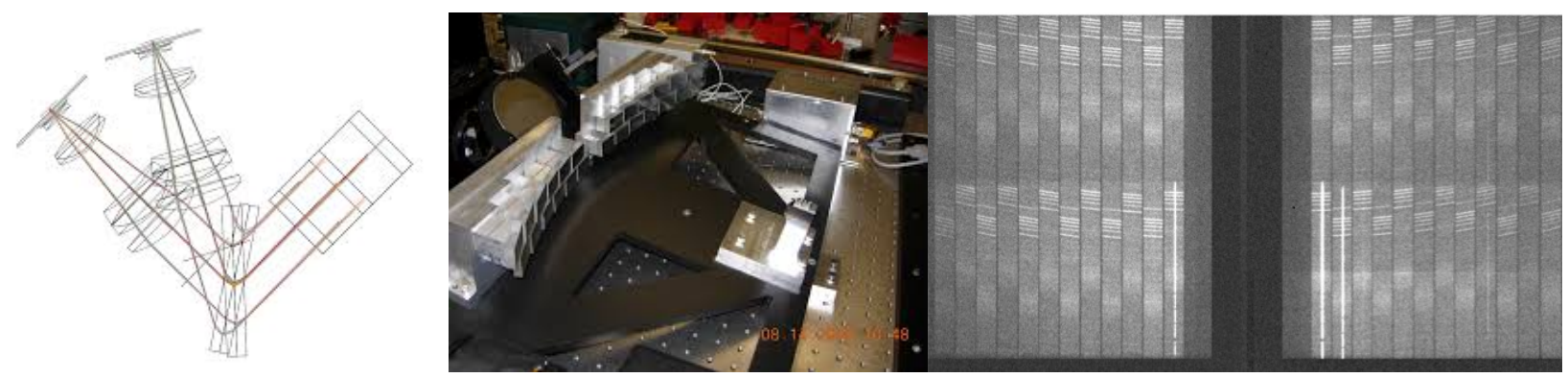

Figure 3. The images on the far left is a schematic showing two grating/camera CCD angles for the CWI high-dispersion grating. ${ }^{23}$ The CWI IFU (middle); the slicer mirrors reflect parts of the telescope focal plane toward the pupil mirror array. The brickwall configuration of the pupil mirrors permits an efficient packing of the spectra on the CCD. The image on the far right is a single nod-and-shuffle frame taken with the CWI CCD. The top half of this image is the background region exposure, a total of 20 minutes, 10 exposures of 2 minutes each. The bottom half of this image is the source region exposure, a total of 20 minutes, 10 exposures of 2 minutes each. ${ }^{22}$

We had requested PCWI observing time to investigate an upgrade of the current CCD to a delta-doped EMCCD. Currently, there is a $50 \%$ loss of time on target due to the "nod and shuffle" of operation. A mask is used to "shuffle" the charge, however, this limits the wavelength range of an observation to an area one third the size of the device. The telescope switches between the object and a nearby sky region at a cadence of $\sim 2$ minutes. As the telescope "nods" between object and sky, the CCD "shuffles" the charge to a region of the CCD under the mask. In this way, readnoise is limited to a single readout every 20 minutes (co-added), while the sky and object integration are excellent matches. This is unavoidable to achieve excellent sky-subtraction. Furthermore, there is a $2 / 3$ loss in wavelength range. One third of the device area is used by the sky, while the remaining third is unused, but necessary for CCD shuffling. Installing an EMCCD as a replacement detector would improve the instrument efficiency by a factor of 3 .

The observing run consisted of an integration of the PCWI control system with Nüvü. This included a computer setup which operates Nüvü, the PCWI grating and camera mechanical control, the telescope guider, temperature monitoring, and $\mathrm{ON} / \mathrm{OFF}$ controller for the lamps and shutter. This integration was smooth and the installation of our dewar and detector system with the spectrograph was a success. It is now a complete working system for any future observations for this on-going project.

Unfortunately, more than $50 \%$ of this time was lost due to high humidity, rain, and cloud cover. In spite of poor observing conditions we acquired some data on a quasar previously observed by CWI. Figure 4 shows the first set of reduced data from this run, and how it compares to the same target taken with the regular CWI CCD. This data is normally reduced via the CWI data reduction pipeline, the EMCCD data has not yet been passed through the pipeline. It must be calibrated correctly before we can compare throughput and performance of both detectors. This data reduction is underway.

\section{FIREBALL-2: FUTURE NOISE GOALS}

These goals are an extension of those presented by Hamden. ${ }^{20}$ FIREBall-2's noise requirements have been designed around a 100 second exposure. Our original aim for this detector was 1000 seconds, however, 


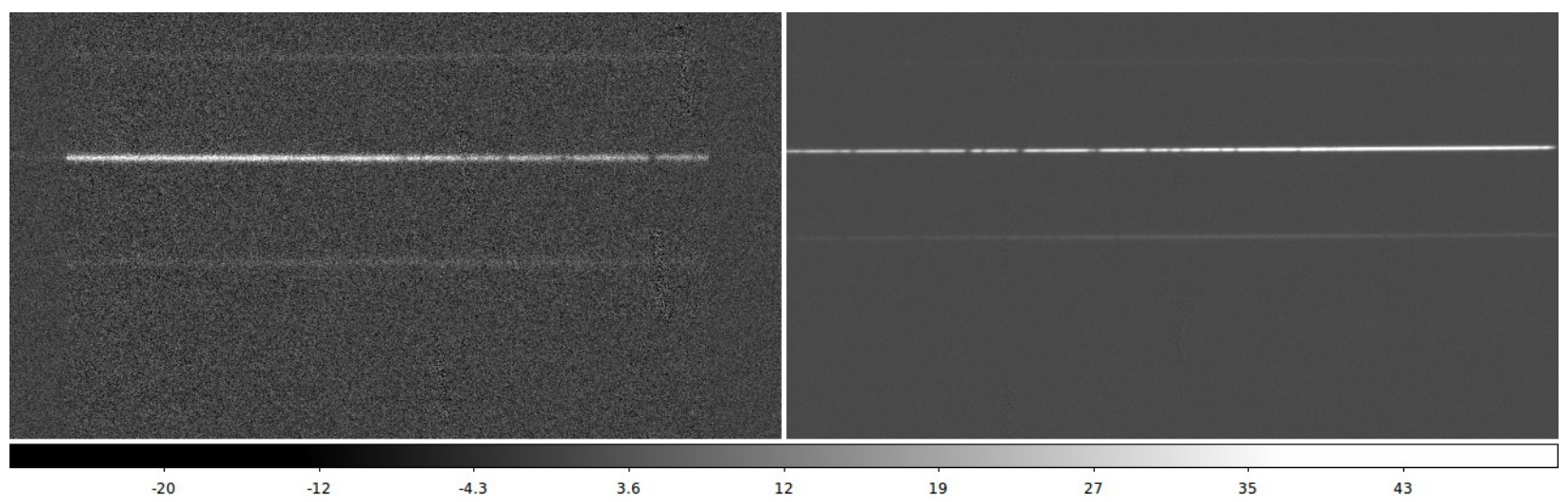

Figure 4. This is an image of QSO1009 obtained using PCWI. The RHS image is a nod \& shuffle image (more detail of this process by Matuszewski ${ }^{22}$ ) of QSO1009 taken in May 2015. It is a 20 minute exposure of the target and is sky-subtracted. The LHS is the same target, observed a year later in 2016. This data was treated similarly to that described in Section 3.2 , where each image is thresholded and finally summed to match the exposure time of the RHS image. Therefore, 200 images at an exposure time of 6 seconds were acquired in photon counting mode (EM gain of 1100e/e).

we have found that due to cosmic-ray rate that this will not be possible. We can still meet our science requirements at this exposure. Most EMCCD applications operate at high frame rates with very fast readout times. ${ }^{9}$ Our work has opened up a new regime in EMCCD spectroscopy in that our primary goal is low dark current for long exposures ( $>100$ seconds). Our preparation in noise testing has led us to set a limit of 0.001 events/pix/frame for both CIC and dark current. Therefore, a total noise limit of 0.002 events/pix/frame in spurious charge. Daigle ${ }^{14}$ discusses a false positive rate of 1 noise event per million pixels assuming a $5 \sigma$ cutoff, a flux rate of 0.1 events/pix/frame, a gain of around 3000 e/e, with a total noise of 0.001 events/pix/frame.

Our total noise during flight operations may be somewhat higher than this, but we expect a low rate of spurious charges per frame. Future testing will focus on dark current performance of delta-doped devices. Furthermore, another goal for this project is a QE measurement with our new clocking configuration, which will be first explored with an engineering grade device as a baseline. A Version 3 Nüvü controller with digital correlated double-sampling, variable clock speeds, and better clocking resolution will assist us in further reduction of noise.

FIREBall-2 is expected to fly in 2017.

\subsection{Acknowledgments}

The research was carried out in part at the Jet Propulsion Laboratory, California Institute of Technology, under a contract with NASA. This work was partially supported by KISS, the W. M. Keck Institute for Space Studies, and by NASA Headquarters under NASA Grant NNX12AF29G.

\section{REFERENCES}

[1] Tuttle, S. E., Schiminovich, D., Milliard, B., Grange, R., Martin, D. C., Rahman, S., Deharveng, J.-M., McLean, R., Tajiri, G., and Matuszewski, M., "The FIREBall fiber-fed UV spectrograph," in [Ground-based and Airborne Instrumentation for Astronomy II], Society of Photo-Optical Instrumentation Engineers (SPIE) Conference Series 7014, 70141T (July 2008).

[2] Milliard, B., Martin, D. C., Schiminovich, D., Evrard, J., Matuszewski, M., Rahman, S., Tuttle, S., McLean, R., Deharveng, J.-M., Mirc, F., Grange, R., and Chave, R., "FIREBALL: the Faint Intergalactic medium Redshifted Emission Balloon: overview and first science flight results," in [Space Telescopes and Instrumentation 2010: Ultraviolet to Gamma Ray], Society of Photo-Optical Instrumentation Engineers (SPIE) Conference Series 7732, 773205 (July 2010). 
[3] Grange, R., Lemaitre, G. R., Quiret, S., Milliard, B., Pascal, S., and Origné, A., "Multi object spectrograph of the Fireball balloon experiment," in [Space Telescopes and Instrumentation 2014: Ultraviolet to Gamma Ray], Society of Photo-Optical Instrumentation Engineers (SPIE) Conference Series 9144, 914430 (July 2014).

[4] Hamden, E. T., Jewell, A. D., Gordon, S., Hennessy, J., Hoenk, M. E., Nikzad, S., Schiminovich, D., and Martin, D. C., "High efficiency CCD detectors at UV wavelengths," in [Space Telescopes and Instrumentation 2014: Ultraviolet to Gamma Ray], Society of Photo-Optical Instrumentation Engineers (SPIE) Conference Series 9144, 91442X (July 2014).

[5] Quiret, S., Milliard, B., Grange, R., Lemaitre, G. R., Caillat, A., Belhadi, M., and Cotel, A., "The FIREBall-2 UV sample grating efficiency at 200-208nm," in [Space Telescopes and Instrumentation 2014: Ultraviolet to Gamma Ray], Society of Photo-Optical Instrumentation Engineers (SPIE) Conference Series 9144, 914432 (July 2014).

[6] Nikzad, S., Hoenk, M. E., Grunthaner, P. J., Terhune, R. W., Grunthaner, F. J., Winzenread, R., Fattahi, M. M., Tseng, H.-F., and Lesser, M. P., "Delta-doped CCDs: high QE with long-term stability at UV and visible wavelengths," in [Instrumentation in Astronomy VIII], Crawford, D. L. and Craine, E. R., eds., Society of Photo-Optical Instrumentation Engineers (SPIE) Conference Series 2198, 907-915 (June 1994).

[7] Nikzad, S., Hoenk, M. E., Greer, F., Jacquot, B., Monacos, S., Jones, T. J., Blacksberg, J., Hamden, E., Schiminovich, D., Martin, C., and Morrissey, P., "Delta-doped electron-multiplied CCD with absolute quantum efficiency over $50 \%$ in the near to far ultraviolet range for single photon counting applications," Applied Optics 51, 365 (Jan. 2011).

[8] Hoenk, M. E., Carver, A. G., Jones, T., Dickie, M., Cheng, P., Greer, F., Nikzad, S., Sgro, J., and Tsur, S., "The duv stability of superlattice-doped cmos detector arrays," in [2013 International Image Sensor Workshop], (June 2013).

[9] Mackay, C. D., Tubbs, R. N., Bell, R., Burt, D. J., Jerram, P., and Moody, I., "Subelectron read noise at mhz pixel rates," Society of Photo-Optical Instrumentation Engineers (SPIE) Conference Series 4306, 289-298 (2001).

[10] Jerram, P., Pool, P. J., Bell, R., Burt, D. J., Bowring, S., Spencer, S., Hazelwood, M., Moody, I., Catlett, N., and Heyes, P. S., "The LLCCD: low-light imaging without the need for an intensifier," in [Sensors and Camera Systems for Scientific, Industrial, and Digital Photography Applications II], Blouke, M. M., Canosa, J., and Sampat, N., eds., Society of Photo-Optical Instrumentation Engineers (SPIE) Conference Series 4306, 178-186 (May 2001).

[11] Gach, J.-L., Guillaume, C., Boissin, O., and Cavadore, C., "First Results of aN L3CCD in Photon Counting Mode," in [Scientific Detectors for Astronomy, The Beginning of a New Era], Amico, P., Beletic, J. W., and Belectic, J. E., eds., Astrophysics and Space Science Library 300, 611-614 (2004).

[12] Tulloch, S. M. and Dhillon, V. S., "On the use of electron-multiplying CCDs for astronomical spectroscopy," Monthly Notices of the Royal Astronomical Society 411, 211-225 (Feb. 2011).

[13] Harpsøe, K. B. W., Andersen, M. I., and Kjægaard, P., "Bayesian photon counting with electronmultiplying charge coupled devices (EMCCDs)," Astronomy \&3 Astrophysics 537, A50 (Jan. 2012).

[14] Daigle, O., Gach, J.-L., Guillaume, C., Lessard, S., Carignan, C., and Blais-Ouellette, S., "CCCP: a CCD controller for counting photons," in [Ground-based and Airborne Instrumentation for Astronomy II], Society of Photo-Optical Instrumentation Engineers (SPIE) Conference Series 7014, 70146L (July 2008).

[15] Tulloch, S., Astronomical Spectroscopy with Electron Multiplying CCDs, PhD thesis, The University of Sheffield (2010).

[16] Daigle, O., Djazovski, O., Laurin, D., Doyon, R., and Artigau, É., "Characterization results of EMCCDs for extreme low-light imaging," in [High Energy, Optical, and Infrared Detectors for Astronomy V], Society of Photo-Optical Instrumentation Engineers (SPIE) Conference Series 8453, 845303 (July 2012).

[17] Daigle, O., Quirion, P.-O., and Lessard, S., "The darkest EMCCD ever," in [High Energy, Optical, and Infrared Detectors for Astronomy IV], Society of Photo-Optical Instrumentation Engineers (SPIE) Conference Series 7742, 774203 (July 2010). 
[18] Nikzad, S., Hoenk, M., Jewell, A. D., Hennessy, J. J., Carver, A. G., Jones, T. J., Goodsall, T. M., Hamden, E. T., Suvarna, P., Bulmer, J., Shahedipour-Sandvik, F., Charbon, E., Padmanabhan, P., Hancock, B., and Bell, L. D., "Single photon counting uv solar-blind detectors using silicon and iii-nitride materials," Sensors 16(6), 927 (2016).

[19] e2v Technologies, Chelmsford, U., "Low-light technical note 4," (April 2015).

[20] Hamden, E. T., Lingner, N., Kyne, G., Morrissey, P., and Martin, D. C., "Noise and dark performance for FIREBall-2 EMCCD delta-doped CCD detector," in [UV, X-Ray, and Gamma-Ray Space Instrumentation for Astronomy XIX], Society of Photo-Optical Instrumentation Engineers (SPIE) Conference Series 9601, 960100 (Sept. 2015).

[21] Janesick, J., [Scientific charge-coupled devices] (2001).

[22] Matuszewski, M., Chang, D., Crabill, R. M., Martin, D. C., Moore, A. M., Morrissey, P., and Rahman, S., "The Cosmic Web Imager: an integral field spectrograph for the Hale Telescope at Palomar Observatory: instrument design and first results," in [Ground-based and Airborne Instrumentation for Astronomy III], Society of Photo-Optical Instrumentation Engineers (SPIE) Conference Series 7735, $77350 \mathrm{P}$ (July 2010).

[23] Martin, C., Moore, A., Morrissey, P., Matuszewski, M., Rahman, S., Adkins, S., and Epps, H., "The Keck Cosmic Web Imager," in [Ground-based and Airborne Instrumentation for Astronomy III], Society of Photo-Optical Instrumentation Engineers (SPIE) Conference Series 7735, 77350M (July 2010). 